\title{
Evaluación del Proceso de Priorización en Salud en Colombia. Perspectiva de Grupos de Investigación Ubicados en Bogotá
}

\section{Evaluating how health is prioritised in Colombia from the point of view of Bogotá-based research groups}

Fabio A. Escobar-Díaz y Carlos A. Agudelo

\begin{abstract}
Universidad Nacional de Colombia, Instituto de Salud pública. faescobard@unal.edu.co, caagudeloc@unal.edu.co
\end{abstract}

Recibido 20 Noviembre 2998/Enviado para Modificación 24 Diciembre 2008/Aceptado 28 Febrero 2009

RESUMEN

Objetivo Evaluar el proceso de priorización de investigaciones en salud llevado a cabo en el país a partir de las metodologías internacionales y desde la perspectiva de los grupos de investigación en salud, categoría A, ubicados en Bogotá.

Métodos: A partir de un enfoque cualitativo, se realizaron 14 entrevistas semies-tructuradas a líderes de los grupos seleccionados a través de una muestra propositiva. Con el programa de análisis de información cualitativa Atlas Ti se generaron categorías para comparación.

Resultados Cada grupo posee diferentes experiencias en investigación en el campo de la salud. Algunos manifestaron sus propias concepciones sobre la salud y sobre la priorización a partir de sus marcos epistemológicos. Diferentes líderes de los grupos expresaron que hay una fuerte orientación biomédica en los procesos de priorización y de las metodologías utilizadas para tal fin. Un número importante de ellos ha reconocido la importancia de la participación de otros actores sociales en la definición de las prioridades para la investigación en salud, además de los mismos investigadores, dentro de un escenario de dialogo y de concertación. Por último, los líderes entrevistados plantearon algunos cuestionamientos frente a la definición de prioridades y sugirieron la importancia de fomentar un proceso más participativo e incluyente comenzando por los mismos investigadores en salud.

Discusión Los hallazgos muestran la enorme heterogeneidad de posiciones frente a la temática de la priorización de investigaciones en salud y las dificultades para alcanzar consensos entre los mismos investigadores.

Palabras Clave: Prioridades de investigación, Prioridades en Salud, Agenda de Investigación en Salud, Política de Investigación en Salud (fuente: DeCS, BIREME) 


\section{ABSTRACT}

Objective Assessing how priorities are established in Colombia in line with international methodologies and from the perspective of Bogotá-based Category A health research groups.

Methods This study used a qualitative approach; 14 leaders from groups selected via a propositive sample were given semi-structured interviews to obtain a compre-hensive interpretation of priority-setting in Colombia. ATLAS Ti software was used for organising information and producing categories from transcripts.

Results Each group had a different research background and came from health research areas such as basic science, clinical science and the wide field of public health. Some talked about their own definitions of health and establishing priorities as related to their own epistemological frameworks. Other leaders stressed that a bio-medical approach still predominated in health research, priority-setting and the inter-national methodologies used for such end. Many recognised the importance of differ-ent social actors (i.e. apart from researchers) becoming involved in defining health research priorities within a scenario emphasising dialogue and coming to agreement. The leaders criticised the national health science and technology system raising questions regarding defining priorities; they stated that dialogue and involvement must be promoted.

Discussion These findings revealed enormous heterogeneity regarding prioritising health research as every researcher has a different point of view due to their experi-ence and backgrounds and the difficulties in researchers' reaching consensus.

Key Words: Priority setting, health research priority, health research agenda. (source: $\mathrm{MeSH}, \mathrm{NLM}$ )

a brecha 10/90 es una expresión que quiere decir que menos del $10 \%$ de los fondos económicos para la investigación en salud en todo el mundo, todos los prove todos los problemas de salud que afecta a la población mundial (1). En el ámbito internacional, el reconocimiento de esta brecha se inició en la década de los años 90 gracias al informe de la Comisión de Investigación en Salud para el Desarrollo-COHRED (1).

De esta forma, los esfuerzos se han realizado principalmente en la identificación de esta brecha en cada una de las naciones, avanzando en políticas y agendas de prioridades para la investigación en salud concertadas con la participación de diferentes actores y sectores sociales que contribuya de forma eficiente y efectiva a resolver los problemas de salud de sus poblaciones.

Se considera que la priorización se relaciona con las decisiones sobre cómo asignar recursos limitados y es una parte fundamental de cualquier proceso de desarrollo de una política de salud. Y, aunque no se cuestiona en absoluto la 
necesidad de establecer prioridades en el contexto de recursos limitados, existen debates tanto teóricos como prácticos acerca de la forma más apropiada para determinar estas mismas prioridades (2). Además, no existe aún el método o el proceso ideal para asegurar la sostenibilidad de la priorización en investigaciones en salud (3). Por lo tanto, se han utilizado diferentes métodos y cada país los ha utilizado según su propio criterio para organizar y definir sus prioridades (4). No obstante, la preocupación por las formas más adecuadas de medición ha marginado temas controversiales pero fundamentales como quién, cómo y qué criterios usar para priorizar investigaciones en salud.

Entre las metodologías internacionales más reconocidas se encuentran: el Enfoque del Consejo de Investigación en Salud para el Desarrollo (COH-RED), el Proceso de los Cinco Pasos el cual fue auspiciado por la Organización Mundial de la Salud y la Matriz Combinada de Aproximaciones o CAM que integra los enfoques anteriores $(5,6)$.

Más allá de las consideraciones metodológicas es esencial tomar en cuenta las circunstancias políticas de los países en donde se pretende establecer este tipo de procesos porque pueden determinar las decisiones para la priorización (3). Y, en segundo lugar, el tema de la participación o involucramiento de diferentes actores, no solo científicos, es fundamental para obtener legitimidad y credibilidad del proceso mediante la participación activa y directa de las organizaciones comunitarias, los formuladores de políticas, las empresas e industrias, y por supuesto los investigadores mismo, conciliando los aspectos técnicos y sociopolíticos en un mismo escenario de debate (3).

Para el caso colombiano, en el reconocimiento de la brecha 10/90 persisten aun desequilibrios. En el año 2004, con la iniciativa de Colciencias y del Instituto de Salud Pública de la Universidad Nacional de Colombia se hizo manifiesto el interés por construir una agenda nacional de prioridades de investigación en salud. Participaron representantes de las comunidades científicas en salud. Se organizaron dos reuniones nacionales, dos reuniones regionales, y un foro virtual. Además, se emplearon las tres metodologías mencionadas anteriormente. La complejidad de las metodologías y del proceso de priorización se hizo evidente en las dificultades que tuvieron algunos investigadores en el uso de los instrumentos diseñados para tal fin (21).

Debido a la relativa novedad de este tipo de procesos en Colombia, son escasos los antecedentes y experiencias previas al respecto. En el plano internacional, son pocas las investigaciones y estudios que aborden y valoren cuál es la 
perspectiva de los investigadores sobre este tipo de procesos, a diferencia de otros abordajes a los pacientes, profesionales e instituciones de salud. Se destacan estudios sobre la capacidad de los países en desarrollo para generar conocimientos e investigación a través de su masa crítica, procesos investigativos y vinculación de otros actores sociales (7), valoración de metodologías hacia profesionales del área clínica (8) y formuladores de políticas (9).

Por consiguiente, este estudio se centró en la evaluación del primer ejercicio de priorización de las investigaciones en salud en Colombia (Ver ensayo sobre el tema en este número). Se buscó reconocer la perspectiva que los grupos científicos tuvieron sobre el proceso, con base en los conocimientos y trayectoria de los investigadores, y a partir también de los modelos internacionales de priorización, pretendiendo con esto establecer un marco analítico que permitiera encontrar significados frente a la priorización y establecer comparaciones entre los diferentes actores abordados.

\section{METODOLOGIA}

Se llevó a cabo un estudio cualitativo descriptivo, mediante el uso de entrevistas individuales semiestructuradas. La población se encuentra conformada por los grupos de investigación en salud clasificados como categoría A por Colciencias. Además, se establecieron como parámetros de selección que estos grupos: a. Debían localizarse en Bogotá, b. Debían estar reconocidos y categorizados por Colciencias como A y c. Representaran los diferentes enfoques de investigación en salud en las áreas básicas, clínicas y salud pública. De esta manera, fueron identificados 43 grupos de investigación clasificados en categoría A en Bogotá.

La muestra cualitativa se caracterizó porque fue definida durante el proceso de recolección y análisis de la información (10), teniendo en cuenta que este tipo de muestreo no pretende la generalización sino reflejar una realidad descubriendo significados múltiples (11). Por lo tanto, se construyó una muestra propositiva que consiste en que el investigador selecciona la muestra más productiva para responder a las preguntas de la investigación en cuanto a la posibilidad de examinar formas de variación y desviación frente al fenómeno estudiado y sujetos con experiencias especiales o específicas sean coincidentes o contradictorios frente al mismo (12). Fueron finalmente seleccionados 14 grupos de investigación en salud categoría A ubicados en Bogotá.

Los grupos de investigación fueron contactados por vía telefónica o correo electrónico para la solicitud de la entrevista. Con el propósito de garantizar el 
mayor rigor metodológico posible, las entrevistas fueron registradas por medio de grabadora de voz, con el previo consentimiento por parte de las personas entrevistadas, se realizaron las transcripciones textuales de las grabaciones (13). Además, el investigador elaboró notas de campo donde consignó las descripciones e interpretaciones sobre la dinámica y el contenido de la entrevista.

Además, tomando como referencia a Strauss y Corbin, se utilizó el criterio de la saturación de categorías (10) como una forma de definir hasta donde continuar el muestreo. En este sentido, se consideró que la saturación aparece cuando no hay datos nuevos que emergen de las categorías. El proceso de análisis e interpretación de los resultados se realizó a partir de estas mismas transcripciones (13). El análisis de la información tuvo una perspectiva inductiva a partir de la descripción y clasificación de la información por medio de categorías o códigos $(10,14)$.

Se empleó el programa Atlas Ti versión 5.0 como herramienta de apoyo para todo el proceso de análisis de la información proveniente de las transcripciones de las diferentes entrevistas. Las transcripciones se realizaron en el programa MS Word y guardados en formato RTF.

Para el proceso de codificación, se inició con la codificación abierta (10), a partir de una lectura inicial de todas las transcripciones de las entrevistas. En esta primera fase surgieron 179 códigos. Posteriormente, buscando progresivamente el refinamiento de las categorías a través de la reconceptualización de la información se fueron reduciendo el número de códigos $(10,14)$. Por lo tanto, se continuó con el proceso de dividir y fusionar categorías para refinarlas, subcategorizarlas y combinarlas (14); así, en una segunda fase de categorización, se redujeron a 35 códigos. En la tercera fase, 25 códigos. Finalmente, quedaron 10 categorías las cuales son descritas en los resultados. Gracias a estas categorías fue posible establecer comparaciones para evidenciar similitudes y al mismo tiempo diferencias en los discursos provenientes de los investigadores e investigadoras entrevistadas alrededor del fenómeno de la priorización de investigaciones en salud (14).

\section{RESULTADOS}

Concepción de Salud. No todos los grupos de investigación comparten una visión homogénea de lo que significa la salud para ellos. Desde esta óptica, surgieron diferentes maneras de comprender esta categoría que influye y determina la comprensión de la priorización como proceso social: "En salud, en el caso que tu estás preguntado pues existen múltiples formas de ver y entender lo que se entiende por salud 
(...) si yo lo que considero es que darles salud a las personas de una nación es construirles hospitales para posibilitar el acceso oportuno o temprano de calidad cuando estén enfermos pues es una visión de salud pero la salud no necesariamente se ve restringida a eso". Otro de los investigadores manifestó que: "si se prioriza con el positivismo la prioridad la hace una técnica y la hace un experto y si una población es evaluada por un experto y por mejores técnicas que técnicas que tenga sin participación, evidentemente ya hay un sesgo de comprensión acerca de la situación que vive la población".

Otros lideres de los grupos de investigación comparten posiciones similares: “... entonces uno sí siente que existe un sesgo hacia un tipo de investigación en específico y que además ese sesgo no necesariamente esta reflejando las verdaderas necesidades de conocimiento que necesita específicamente, necesitamos en salud en el país”;“'Inclusive la misma manera como se presentan los proyectos no le permiten la investigación social que le gusta trabajar en medicina, no puede presentar proyectos porque es que no son métodos cuantitativos".

Priorización como concepto. Quienes desconocen la priorización desarrollaron una conceptualización espontánea con base en criterios que se derivan de su propia experiencia investigativa: "Cómo no se qué es un proceso de priorización, es decir yo lo hablo desde mi intuición de lo que significa la palabra priorizar que es poner en una escala lo que se considera más importante y darle digamos vía libre a los mas importantes, eso es lo que yo llamaría priorizar"; "sería como un proceso en el que uno jerarquiza, identifica qué tiene más relevancia con respecto a otras cosas y esa se le da pues más importancia y pues se prioriza".

Sin embargo otros grupos desarrollaron una conceptualización mas detallada de la priorización: "Priorizar en investigaciones en salud es como fijar una, un escalafón digamos que pueda servir para finalmente para saber en primer lugar cuáles son las necesidades de investigación del país y clasificarlas de acuerdo con su importancia, teniendo en cuenta especialmente por ejemplo el perfil epidemiológico, la importancia y el impacto que ellos tienen en los indicadores de carga de enfermedad por ejemplo, etcétera, etcétera, y con base en esa clasificación ya poder decir que se van a destinar, o sea, poder destinar recursos en consecuencia o en consonancia con la fijación de esas prioridades".

Priorización como Experiencia. Algunos de los lideres destacaron el desconocimiento que tienen sobre experiencias relacionadas con la priorización: "no conozco, no quiere decir que no exista pero no lo conozco ni he participado"; "taller como tal en que se siente uno tres, cinco días a mirar qué es la priorización en salud cómo debemos enfocar esto, cómo vamos a asumir los retos, jamás”

Otros grupos mencionaron de una manera explicita la experiencia realizada por Colciencias y la Universidad Nacional en donde participaron: "conocimos el ejercicio que Colciencias contrató con la Universidad Nacional y digamos que en ese sentido pues pudimos ver el grupo de personas que habían sido involucradas en el proceso de priorización". 
En cuanto a experiencias internacionales, algunos grupos destacaron: "sí conozco algunos en Latinoamérica de priorización en salud, incluso en Iberoamérica porque conozco tres que se han hecho, uno en España, uno en Argentina y uno en Perú. Son talleres, o sea, conozco los que...que me han comentado es en modalidad de taller con participación interdisciplinaria y con participación también estatal, es decir, el estado, la academia y dentro de la academia, varias disciplinas" Otro de los investigadores resaltó el proceso de priorización realizado en Canadá: "Lo que uno diría de la experiencia canadiense es que ellos hacen un ejercicio a partir de primero los problemas de salud, y partir de los problemas de salud identifican cuáles son los interrogantes que están relacionados con estos problemas de salud y partir de estos interrogantes entonces definen, si, líneas de investigación”.

Experiencias en Metodologías de Priorización. Algunos de líderes entrevistados quienes nunca han participado en este tipo de experiencias dijeron: "Se que existen pero las guías como tal no las se, no las conozco"; "No, así como que nos haya llegado, no. Más bien lo haríamos como intuitivamente o por necesidad".

Por otra parte, uno de los lideres reconoció las metodologías internacionales que se utilizan en la construcción de prioridades para la investigación en salud: "EI que hizo LATINCLEN se basó fundamentalmente en el COHRED en el del consejo para investigación esencial en salud"; "el de Colciencias si no estoy equivocado se basó mas en la metodología que tiene el Global Forum for Health Research".

Otro de los investigadores también ha destacado esta metodología como la más notable dentro de la experiencia de Colciencias: "en la primera reunión nos presentaron como las metodologías especialmente en lo que tiene que ver con el, con lo recomendado por el Foro Mundial de Investigación en Salud, el Global Forum for Health Research, y ellos tienen como una metodología recomendada para eso y creo que esa fue la que se siguió finalmente".

Criterios para definir la prioridad. Esta categoría es bastante controversial para los grupos porque no hay una homogeneidad de criterios con respecto a la forma en que debe definirse una prioridad para la investigación en salud. Cada grupo generales, considera que su área de investigación es prioritaria, así para el Sistema Nacional de Ciencia y tecnología no sea así. Por ejemplo, para el caso del estudio de la urticaria papular por picadura de pulga: "Prioritario desde el punto de vista mío porque es un motivo de consulta muy frecuente en Bogotá y en las áreas de clima frío". Sin embargo, como la líder de este grupo de investigación agregó: "yo solamente he tenido una vez...de financiación por Colciencias porque ellos no consideran que es una línea de impacto en salud, si, porque consideran que la alergia a la picadura de pulga porque como uno no se muere de eso, si, entonces no es importante".

De manera muy similar a esta argumentación, la representante de otro de los grupos de investigación, relacionado con el campo de la salud oral, afirmó que: "la 
odontología pues no cabe ni entra ni nada porque no mata, si, a no ser que nos metamos pues con la cardiovascular o con el cáncer oral".

Algo similar piensan los lideres de otros grupos: "si usted mira ahora las neurociencias o los problemas del sistema nervioso central eso no figura en las prioridades del ministerio ni de Colciencias", y desde el punto de vista de la genética: "uno puede decir bueno lo prioritario son los problemas de salud que afectan a la población no, entonces, malaria es un problema muy complicado en Colombia, la fiebre amarilla es un problema muy complicado, la hepatitis es un problema muy complicado entonces esas son las prioridades, pero un minuto, ¿las prioridades no son por ejemplo que miremos desde la Genómica como entender eso?".

Espacialidad de la Priorización. Se hace referencia a los elementos territoriales, regionales y locales que pueden configurar la definición de una agenda de prioridades para la investigación en salud. Uno de los investigadores entrevistados afirmó que: "hay que entender la especificidad de cada uno de los contextos en términos también de que pueden ser investigaciones que no tienen un peso importante a nivel agregado pero que pueden ser muy importantes en la especificidad de ese contexto que usted está viviendo". Además, otro de los investigadores entrevistados aseguró que es importante: "saber quién es fuerte en qué región y cómo la región se beneficia de tener grupos en tal cosa, o cuál es el potencial en términos de desarrollo científico tecnológico de la Costa Atlántica, y cómo los Llanos pueden perfectamente beneficiarse y no simplemente repetir la misma cosa siete veces en diferentes regiones de Colombia".

Temporalidad de la Priorización. Se ha considerado que la priorización implican procesos que deben ser formulados y reformulados durante ciertos periodos de tiempo debido a que las condiciones y contextos que constituyen la fuente de estas prioridades van cambiando. Uno de los actores entrevistados aseguró que: "todos estos procesos son en cierta medida de nunca acabar porque las condiciones cambian y al cambiar las condiciones puede que las prioridades cambien, entonces el hecho de que se haya hecho un ejercicio diez años atrás no quiere decir que esas prioridades establecidas diez años atrás se van a mantener por los próximos cincuenta, muy seguramente no es así".

De acuerdo con otro de los investigadores, una visión estratégica sobre los problemas de salud que se están investigando permite una mayor capacidad de anticiparse y prevenir situaciones futuras: "la visión estratégica es fundamental porque usted puede prevenir y darle una mejor solución a lo que se va a volver prioridad en el futuro porque si no, usted va a la cola de los acontecimiento".

Dialogo participativo intersectorial. La mayoría de grupos entrevistados están de acuerdo en que la priorización de investigaciones en salud implica la participación y la interacción entre los mismos investigadores y de estos con la 
sociedad en su conjunto. Al respecto señalaron ideas como: "yo creo que es necesario un proceso en que se sienten a hablar los diferentes actores para poder organizar una priorización en investigaciones en salud". Para otro investigador: "yo pienso que es una construcción colectiva de la prioridad porque lo que se plantea es un debate alrededor de qué es mas relevante o no en términos de las relaciones causales y digamos no se hace desde una fortaleza individual dentro [...], sino que se trata de hacer un debate o una construcción".

Los investigadores deben ser protagonistas de estos procesos pero no los únicos, tal como lo señaló uno de los lideres: "es importante que los grupos sean reconocidos pero no que se conviertan en los únicos actores que pueden hablar en términos de las investigaciones que se deben desarrollar". Esto es compartido por otro de los lideres entrevistados: "no debe ser un ejercicio que se le deje exclusivamente a los investigadores, mucho menos si el proceso se establece por ejemplo para definir las prioridades de un país o de una ciudad".

Priorización excluyente. Cuando se establecen prioridades algunos investigadores perciben que existe el riesgo de que, debido a los recursos económicos limitados para financiar proyectos, sus áreas de investigación no sean incluidas o queden relegados definitivamente por fuera de las prioridades y perjudique su trabajo investigativo. Por ejemplo: "la priorización, dependiendo de los problemas de salud en Colombia, deja por fuera una cantidad enorme de gente bien entrenada, no, entonces tradicionalmente malaria, chagas, se han comido una cantidad enorme de presupuesto de investigación en Colombia, son problemas muy complicados de obviamente de salud publica en nuestro medio pero hay una gran cantidad de otras áreas de investigación que no están representadas en problemas importantes de salud publica aparentemente sobre los cuales hay una cantidad enorme de gente bien preparada, calificada, competitiva de modo que el proceso de priorización es un proceso per se complejo".

Valoración del Sistema Nacional de Ciencia y Tecnología en Salud. Este es otro de los temas más controversiales y donde se han expresado diferentes posiciones. Uno de los investigadores señaló: "hay unas prioridades que fijó Colciencias, pero no están directamente ligadas con los problemas de salud digamos más sentidos por la comunidad".

Para otra investigadora, existe también inconformidad sobre el papel de Colciencias en su relación con los grupos de investigación: "Pues lo que ellos tienen es que tener muy claro en qué está trabajando la gente, cuántos grupos tienen registrados, para ver si las políticas que ellos tienen están cubriendo realmente lo que todo el mundo está trabajando para que eso no solamente cubriera unos pocos y dependiendo de lo que la gente está trabajando". Otra investigadora ha señalado la ausencia de indicadores apropiados para definir las prioridades de investigación en salud en el país: "varios directores de Colciencias que han venido y han hablado con nosotros, cuando uno les pregunta cómo han sido los indicadores para priorizar líneas, quedan locos, no nos pueden decir cuáles fueron los indicadores, o sea, no hay unos indicadores o unos instrumentos, si, sensibles y válidos para determinar cuáles deben ser las líneas prioritarias para el país". 
De acuerdo a otro de los investigadores entrevistados, el papel de Colciencias debe ser independiente de los intereses de los ministerios de turno: "Colciencias debería impulsar la ciencia y la investigación, claro, con las mismas limitantes, pero no puede ser un apéndice del ministerio de salud". Además, se mencionó otra dificultad que se presenta en las convocatorias de Colciencias y que se relacionan con las prioridades que define la institución para la investigación: "la gente cuando pasa un proyecto a Colciencias termina viendo a ver si su trabajo se puede articular a las políticas que le señala Colciencias para apoyar proyectos y entonces termina es priorizando de acuerdo con eso".

Uno de los lideres de los grupos añadió que: "hay unos intereses prioritarios detrás, que salen seguramente del Consejo Nacional de Ciencia y Tecnología hoy en día mucho mas que la discusión entre todos nosotros los investigadores que hace tiempo no se da".

La investigadora líder de otro de los grupos de investigación también manifestó una posición crítica frente a Colciencias: "yo creo que personalmente por lo que he vivido o sea a veces como que Colciencias le da toda la financiación a un mismo grupo, si, entonces son diez personas del mismo grupo distribuidos en [...] toda la financiación que tiene es a ese grupo, y resulta que hay gente de diferentes departamentos hay gente de diferentes áreas, hay gente que son muy pilos también de otras universidades". Otro de los investigadores ha planteado también algo similar en el sentido de que Colciencias mantiene el mismo esquema en la asignación de recursos para la investigación, reproduciendo lo mismos errores en términos de las prioridades: "si tu miras los resultados que salieron de Colciencias hace dos días de los anteproyectos que se presentaron, uno nuevamente los mira y ve lo mismo de los años anteriores, si, es una colcha de retazos que uno no encuentra cuáles son las prioridades de investigación del país".

\section{DISCUSIÓN}

Cada uno de los grupos de investigación entrevistados proviene de diversos enfoques metodológicos y epistemológicos y pertenecen a los campos de la ciencia básica, clínica, epidemiología, salud pública y ciencias sociales. En otras palabras, los resultados muestran la gran heterogeneidad que conforma la investigación en salud a través de los aspectos biológicos, psicológicos, sociales y culturales que la caracterizan (15). Paralelo al paradigma positivista, por lo tanto, han surgido enfoques o modelos holísticos, donde se hace un mayor esfuerzo por articular los procesos biológicos con los sociales (16), y donde el ser humano es comprendido como un todo en donde múltiples factores interrelacionan entre si, desde un punto de vista no mecanicista sino multicausal y donde la enfermedad no está aislada de su contexto social (17).

Se ha sugerido que en el campo de la salud pública, más que buscar la complementariedad de metodologías, se necesita establecer aproximaciones entre 
las diferentes posturas epistemológicas para lograr un verdadero enfoque holístico del proceso de la salud y la enfermedad humanas que sea integradora (15). Algunos de los líderes reclamaron la importancia de vincular determinantes sociales, económicos, políticos y culturales de las enfermedades, destacando el fuerte predominio de la investigación básica y clínica en el campo de la salud $(18,19)$. Otros destacaron sus propias áreas de estudio como prioritarias o como estratégicas en términos del perfil epidemiológico actual o futuro del país, resaltando la inquietud de ser excluidos de las prioridades si se optan por criterios poco incluyentes y limitados a ciertas áreas o problemas de salud.

La mayoría de grupos coincidieron en afirman el carácter temporal y espacial de la priorización de investigaciones en salud, señalando su dinámica propia y flexible de acuerdo a la realidad regional y local y sus transformaciones a través del tiempo de acuerdo a los cambios que se presenten en las condiciones y determinantes de los eventos de salud y de enfermedad.

Otro punto de coincidencia fue el carácter participativo e intersectorial de este tipo de procesos. Se planteó la necesidad de una mayor vinculación tanto de los mismos investigadores como de otros sectores sociales en la definición de una agenda de prioridades. La experiencia realizada por Colciencias fue un ejercicio donde sólo participaron algunos investigadores en diferentes áreas del conocimiento y funcionarios provenientes de instituciones estatales como el Ministerio de la Protección Social y algunas Secretarías de Salud (21). Sin embargo, hay experiencias internacionales como la de Brasil y la de Filipinas que han mostrado la importancia de un proceso colectivo y participativo a pesar de la complejidad que implica el mismo (20).

Por último, la valoración que hicieron los grupos entrevistados del Sistema Nacional de Ciencia y Tecnología genera inquietudes que deben ser examinadas con mayor detalle en otros estudios o análisis. Teniendo en cuenta que este estudio no pretende generalizar sus resultados a todo el conjunto de grupos de investigación del país, debe considerarse los planteamientos brindados por los líderes abordados. La relación del SNCTS con todos los grupos y la definición de prioridades de investigación en salud son dos elementos que deben ser sometidos a la discusión en aras de fortalecer el sistema de investigación en salud colombiano de acuerdo a las necesidades de conocimiento y de salud. 
Los resultados de este estudio, a pesar de sus limitaciones en términos de la generalización de los hallazgos a toda la comunidad de investigadores en salud, genera aportes valiosos para la construcción de una agenda de prioridades de investigación en salud en el país, que cuente con la participación concertada de los diferentes actores sociales y para el fortalecimiento del Sistema Nacional de Ciencia y Tecnología en Salud •

\section{REFERENCIAS}

1.Davey S (Ed). The 10/90 report on health research 2003 - 2004. Ginebra: Global Forum for Health Research, 2004. [Internet]. Disponible en: www.globalforumhealth.org. Acceso: agosto 12 de 2007.

2.Reichenbach $\mathrm{L}$. The politics of priority setting for reproductive health: breast and cervical cancer in Ghana. Reproductive health matters 2002; 10 (20): 47-58.

3. COHRED. Priority setting for health research: toward a management process for low and middle income countries. Working paper 1. 2006. [Internet]. Disponible en: http:// www. HealthResearchForDevelopment.org/ Acceso Julio 24 de 2007.

4. Haan S, Montorzi G. Priority setting for health research: toward a management process for low and middle income countries. Well defined management and performance processes help bring national plans of life. CORHED, annual report 2005. [Internet]. Disponible en: http://www.HealthResearchForDevelopment.org/ Acceso:Julio 24 de 2007.

5. The Collaborative Training Program. Health Research for Policy, Action and Practice. Training Modules. The Collaborative Training Program for Health Research for Policy, Action and Practice, Version 1; 2002

6.Okello D, Chongtrakul P. Un Manual para el Establecimiento de Prioridades de Investigación usando la estrategia INES (ENHR). Consejo de Investigación en Salud para el Desarrollo. Documento del COHRED 2002.2, julio de 2002. [Internet]. Disponible en: http:// www. HealthResearchForDevelopment.org/ Acceso: Agosto 24 de 2008.

7. Gonzalez Block MA, Mills A. Assessing capacity for health policy and system research in low and middle income countries. Health Research and Policy Systems 2003, 1:1. [Internet]. Disponible en: http://www.health-policy-systems.com/content////l. Acceso: Agosto 24 de 2008

8. Mitton CR, Donaldson C. Setting priorities and allocating resources in health regions: lessons from a project evaluating program budgeting and marginal analysis (PBMA). Health Policy 2003, 64: 335-348.

9. Kapiriri L, Frithjof $O$, Heggenhougen K. Using burden of disease information for health plan-ning in developing countries: the experience from Uganda. Social Science and Medicine 2003; 56: $2433-2441$.

10. Strauss A, Corbin J. Bases de la investigación cualitativa. Técnicas y procedimientos para desarrollar la teoría fundamentada. Medellín: Editorial Universidad de Antioquia;2002.

11. Martín-Crespo MC, Salamanca-Castro AB. El muestreo en la investigación cualitativa. En: Nurs Investigación. 2007; 27 ( 27).

12. Marshall MN. Sampling for qualitative research. Family Practice. 1996; 3 (6): 522-525.

13. Castillo $E$, Vásquez ML. El rigor metodológico en la Investigación cualitativa. Colombia Médica 2003; 34 (3): 164-167.

14. Dey I. Qualitative Data Analysis: a user friendly guide for social scientists. London: Routledge;1996.

15. Idrovo AJ. Hacia una Salud Pública Pluralista: El Caso de los Plaguicidas y la Salud Humana. Rev. Salud Pública, 2005; 7 (3): 349 - 359.

16. Krieger $\mathrm{N}$. Theories for social epidemiology in the $21^{\text {st }}$ century: an ecosocial perspective. Int $\mathrm{J}$ Epidemiol 2001; 30: 668-677.

17. Bonilla JC. La Crisis Epistemológica y las Ciencias de la Salud. Hacia la Promoción de la Salud, 2004; $9: 7-15$. 
18. Baum F. Changing global research agenda: a call for a greater focus on the social, eco-nomic and political determinants of health. Global Forum for Health Research. Forum 8, Mexico City, November 2004. [Internet]. Disponible en: http://www.globalforumhealth.org/Forum8/ Forum8CDROM/OralPresentations/BaumFF8-590.doc Acceso: agosto 10 de 2008.

19. Breilh J. Methodological Innovation, intercultural knowledge building and Empowerment: The case of participatory research in an agro industrial region. Global Forum for Health Research. Forum 8, Mexico City, November 2004. [Internet]. Disponible en: http:/www.globalforumhealth.org/ Forum8/Forum8-CDROM/OralPresentations/BreilhJF8-143.doc Acceso: Agosto 15 de 2008.

20. Feranil AB. The Buttom Up Approach in Research Agenda Setting: Lessons from the Philip-pine Experience. [Internet]. Disponible en: http://www.globalforumhealth.org/Forum8/Forum8CDROM/OralPresentations/FeranilAF8-219.doc Acceso Agosto 22 de 2008.

21. Construcción de una agenda de priorización de investigación en salud. Memorias. Instituto de Salud Publica, Colciencias, Global Forum for Health Research. Febrero 23 a 25 de 2008. [Internet]. Disponible en: http://www.revmed.unal.edu.co/red/prioridad/memorias.htm Acceso mayo 30 de 2007. 